\title{
The metabolism of high intakes of tryptophan, nicotinamide and nicotinic acid in the rat
}

\author{
BY GWYN M. MCCREANOR* AND DAVID A. BENDER \\ Courtauld Institute of Biochemistry, The Middlesex Hospital \\ Medical School, London WIP $7 P N$
}

(Received 24 February 1986 - Accepted 15 July 1986)

\begin{abstract}
1. The metabolic fate of high dietary intakes of nicotinamide, nicotinic acid and tryptophan, and of acute doses of nicotinamide and nicotinic acid, has been studied in the rat. A new high-pressure liquid chromatography method for measurement of the principal urinary metabolites of niacin is described.

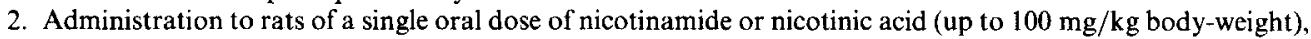
or maintenance for 3 weeks on diets providing $150 \mathrm{mg}$ nicotinamide or nicotinic acid $/ \mathrm{kg}$ diet, resulted in only a small increase in the liver content of nicotinamide nucleotide coenzymes (NAD and NADP). The quantitative metabolism of nicotinamide and nicotinic acid differed, suggesting that intestinal bacterial deamidation is not the major fate of nicotinamide.

3. A high dietary intake of tryptophan $(5.9 \mathrm{~g} / \mathrm{kg}$ diet) led to a considerable increase in liver NAD(P) and also in urinary excretion of niacin metabolites. The results suggest that, as indicated by enzyme kinetic studies (Bender et al. 1982), the utilization of nicotinamide and nicotinic acid for nucleotide synthesis is limited, while there is little or no limitation of NAD(P) synthesis from the tryptophan metabolite quinolinic acid.
\end{abstract}

The established physiological function of niacin is as a precursor of the nicotinamide nucleotide coenzymes, NAD and NADP. The conventional view is that tryptophan can 'substitute' for niacin when the dietary intake of the vitamin is inadequate, since the amino acid can give rise to the nicotinamide nucleotides by way of quinolinic acid, an intermediate in the oxidative pathway of tryptophan metabolism (see Fig. 1).

There are several clinical conditions in which pellagra results from disturbed tryptophan metabolism despite an apparently adequate intake of niacin. Examples include Hartnup disease (Baron et al. 1956), carcinoid syndrome (Castiello \& Lynch, 1972; Lehman, 1972; Swain et al. 1976), the treatment of tuberculosis with isoniazid (Biehl \& Vilter, 1954; Harrison \& Feiwel, 1956) and a recently described hereditary pellagra-like condition which did not respond to the administration of nicotinamide (Salih et al. 1985). All these findings suggest that under normal conditions tryptophan may be more important as a precursor of the nucleotides than is preformed dietary niacin. A recent re-examination of the diets of American pellagrins during the 1930s and 1940s by Carpenter \& Lewin (1985) confirms this view. They calculated that the average intake of available niacin equivalents was apparently adequate in most cases of confirmed pellagra, and concluded that 'the pellagra epidemic in the USA in the early 1900s has not yet been explained satisfactorily'. A previous study from this laboratory (Bender et al. 1982) suggested, largely on the basis of enzyme kinetic studies, that the utilization of nicotinamide and nicotinic acid for nucleotide synthesis was strictly limited, while there was little or no limitation of NAD(P) synthesis from the tryptophan metabolite quinolinic acid.

Very high intakes of niacin, many times larger than the conventionally accepted vitamin levels, are used clinically: nicotinic acid as a hypolipidaemic-hypocholesterolaemic agent (Parsons, $1961 a$ ), and nicotinamide in the treatment of schizophrenia (Osmond \& Hoffer, 1962; Hoffer, 1971) and other psychiatric disorders (Blom et al. 1985). It is often assumed

* Present address: Division of Inherited Metabolic Diseases, MRC Clinical Research Centre, Harrow, Middlesex HAl 3UJ. 


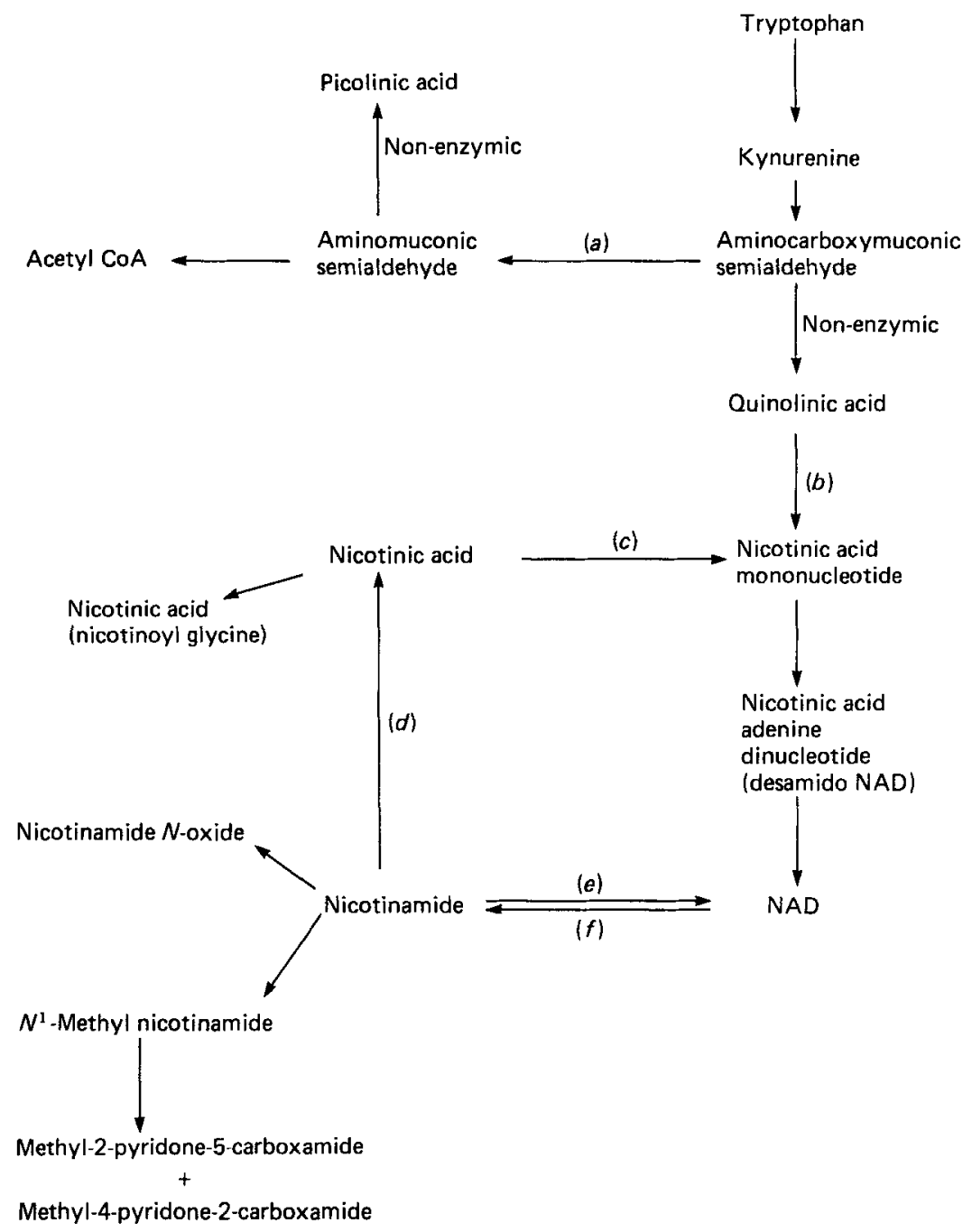

Fig. 1. Pathways of nicotinamide nucleotide coenzyme synthesis and catabolism. (a), Picolinate carboxylase (aminocarboxymuconate-semialdehyde decarboxylase, EC 4.1.1.45); (b), quinolinate phosphoribosyltransferase (nicotinate nucleotide pyrophosphorylase (carboxylating), EC 2.4.2.19); (c), nicotinate phosphoribosyltransferase $(E C 2.4 .2 .11) ;(d)$, nicotinamide deamidase (nicotinamidase, $E C$ 3.5.1.19); (e), nicotinamide phosphoribosyltransferase $\left(E C\right.$ 2.4.2.12); $(f) \mathrm{NAD}^{+}$nucleosidase $(E C 3.2 .2 .5)$ and poly-(ADP-ribose) synthetase (NAD ${ }^{+} \mathrm{ADP}$-ribosyltransferase, $E C$ 2.4.2.30).

that like the other water-soluble vitamins, niacin is not toxic, since it is readily metabolized and excreted. However, in addition to the acute toxicity of single large doses of nicotinic acid (Bartlett et al. 1982), there is evidence of hepatotoxicity associated with the intake of relatively large amounts of both nicotinic acid (Parsons, $1961 a, b$; Baggenstoss et al. 1967) and nicotinamide (Winter \& Boyer, 1973; Kang-Lee et al. 1983).

The present study was undertaken in order to investigate further the relative importance of tryptophan and preformed dietary nicotinic acid and nicotinamide as precursors of the nicotinamide nucleotide coenzymes, and to investigate the metabolic fate of large amounts of niacin. 


\section{METHODS}

Animals and diets

Male Wistar rats bred in the Courtauld Institute of Biochemistry were used. For the acute studies they were maintained on standard animal house diet (diet 86; A. Dixon \& Sons, Ware, Herts) for 3 weeks after weaning. According to information provided by the manufacturers, this diet provided $2.4 \mathrm{~g}$ tryptophan and $73 \mathrm{mg}$ nicotinic acid $/ \mathrm{kg}$. The rats received a single dose of 1,10 or $100 \mathrm{mg}$ nicotinic acid or nicotinamide $/ \mathrm{kg}$ body-weight, dissolved in saline ( $0.15 \mathrm{M}$-sodium chloride; $0.5 \mathrm{ml}$ per animal), by oral intubation. Control animals received $0.5 \mathrm{ml}$ saline alone. They were then housed in individual stainless steel metabolism cages (Acme Metal Co, Chicago, Ill) for $24 \mathrm{~h}$, to permit collection of urine. During this period they were allowed free access to water, but were deprived of food. Each $24 \mathrm{~h}$ collection of urine was diluted to $20 \mathrm{ml}$ with distilled water, then frozen and stored at $-20^{\circ}$ until required.

For the diet studies, animals were weaned on to the experimental diets $21 \mathrm{~d}$ after birth, and were maintained on them for 3 weeks, after which they were housed in metabolism cages for $24 \mathrm{~h}$ for collection of urine, as described previously. They were allowed ad lib. access to food and water; the food intakes shown in Table 3 (p. 582) were determined during this period. The diets used were prepared as described previously (Bender et al. 1982), and were a modification of the maize-gelatine-sucrose diet described by Carter et al. (1977). Six different diets were used, as shown in Table 3. Two diets provided no preformed niacin apart from that present in the maize meal, with an adequate $(1.9 \mathrm{~g} / \mathrm{kg})$ or more than adequate $(5.9 \mathrm{~g} / \mathrm{kg})$ amount of tryptophan. The other four diets provided a minimum amount of tryptophan, determined previously to be adequate to permit limited growth of weanling rats (Magboul \& Bender, 1983), with either nicotinic acid or nicotinamide added at $15 \mathrm{mg} / \mathrm{kg}$ ((US) National Research Council (1962) recommended amount for the rat) or $150 \mathrm{mg} / \mathrm{kg}$.

\section{Analytical methods}

The rats were killed by cervical dislocation and a portion of the liver (about $1 \mathrm{~g}$ ) was rapidly dissected out, frozen in liquid nitrogen and used for measurement of total nicotinamide nucleotides (NAD $(\mathrm{P})^{+}$and $\left.\mathrm{NAD}(\mathrm{P}) \mathrm{H}\right)$, by the fluorimetric method described previously (Bender et al. 1982); a modification of the methods described by Kaplan et al. (1951) and Lowry et al. (1961).

$N^{1}$-Methyl nicotinamide was measured by the small-scale modification of the alkaliketone fluorimetric method of Carpenter \& Kodicek (1950) described previously (Bender, 1980).

Methyl-2-pyridone-5-carboxamide was synthesized by the method of Holman \& Wiegand (1948); other niacin metabolites were purchased from Sigma (London) Chemical Co Ltd, Poole, Dorset.

High-pressure liquid chromatography $(H P L C)$ determination of urinary niacin metabolites The samples were desalted by the addition of $15 \mathrm{ml}$ acetone to $5 \mathrm{ml}$ diluted urine. After standing at room temperature for $30 \mathrm{~min}$, precipitated salts were removed by centrifugation at $2000 \mathrm{~g}$ for $20 \mathrm{~min}$, and the acetone was removed by extraction twice with $5 \mathrm{ml}$ chloroform. Of the resultant aqueous phase, $2 \mathrm{ml}$ was mixed with $0.2 \mathrm{ml} 2.5 \mathrm{M}$-ammonium hydroxide, and was loaded on to a column of Dowex $1 \mathrm{Cl}^{-}$ion-exchange resin. The columns of resin were prepared by pouring an aqueous slurry of resin into a Pasteur pipette plugged at the constriction with glass wool, and each contained $300 \mathrm{mg}$ dry weight of resin. The columns were washed with $10 \mathrm{ml} 2 \mathrm{M}$-hydrochloric acid, followed by $10 \mathrm{ml}$ distilled water, before the application of the sample. 
Table 1. Retention times of tryptophan and niacin metabolites on high pressure liquid chromatography (HPLC)

(HPLC was performed using a $250 \mathrm{~mm} \times 5 \mathrm{~mm}$ column of Partisil 10-SCX cation-exchange resin, eluted isocratically at $1.5 \mathrm{ml} / \mathrm{min}$ with $0.05 \mathrm{M}$-sodium phosphate buffer, $\mathrm{pH} 1.7$, containing methanol $(100 \mathrm{ml} / 1))$

\begin{tabular}{lcc}
\hline \multicolumn{1}{c}{ Compound } & $\begin{array}{c}\text { Retention time } \\
\text { (min) }\end{array}$ & $\begin{array}{c}\text { Fraction from } \\
\text { Dowex 1 column }\end{array}$ \\
\hline iso-Nicotinic acid & $4 \cdot 3$ & Acidic \\
Nicotinamide $N$-oxide & $4 \cdot 4$ & Neutral \\
Methyl-2-pyridone-5-carboxamide & $5 \cdot 2$ & Neutral \\
Picolinic acid & $5 \cdot 3$ & Acidic \\
Nicotinic acid & $5 \cdot 8$ & Acidic \\
Nicotinuric acid & $7 \cdot 7$ & Acidic \\
Unidentified peak (tentatively assumed to be & $8 \cdot 0$ & Neutral \\
$\quad$ methyl-4-pyridone-2-carboxamide) & & Neutral \\
Nicotinamide & $11 \cdot 7$ & Neutral \\
\hline
\end{tabular}

The initial eluate from the column and that from washing with $8 \mathrm{ml}$ water were collected; neutral compounds (nicotinamide, nicotinamide $N$-oxide, $N^{1}$-methyl nicotinamide and methyl-2-pyridone-5-carboxamide) were in this fraction. Acidic compounds (nicotinic and nicotinuric acids) were then eluted with $4 \mathrm{ml} 1 \mathrm{M}-\mathrm{HCl}$. The eluates were evaporated to dryness in a vortex evaporator under reduced pressure at $60^{\circ}$, and were then redissolved in $0.2 \mathrm{ml} 0.05 \mathrm{M}$-sodium phosphate buffer, $\mathrm{pH} 1.7$, containing methanol $(100 \mathrm{ml} / \mathrm{l})$. In order to determine whether any of the unidentified peaks that were detected by HPLC were due to glucuronide or sulphate conjugates, samples of the urine extract prepared for HPLC were incubated with 1000 units of a preparation of $\beta$-glucuronidase $(E C 3.2 .1 .31)$ from Helix pomatia (Sigma (London) Chemical Co Ltd), containing 50 units of sulphatase activity, at $\mathrm{pH} 5.0$ and $30^{\circ}$ for $60 \mathrm{~min}$.

Preliminary studies showed that the initial separation into acidic and neutral fractions before HPLC was necessary because picolinic acid, a metabolite of tryptophan which elutes in the acidic fraction, had the same retention time on HPLC (5.3 min) as methyl2-pyridone-5-carboxamide $(5 \cdot 2 \mathrm{~min})$, which elutes in the neutral fraction. Similarly, isonicotinic acid, which was added to samples as an internal standard, overlapped with nicotinamide $N$-oxide unless they were first separated by ion-exchange chromatography (see Table 1).

HPLC was carried out using a Partisil 10-SCX cation-exchange column $(250 \mathrm{~mm} \times 5 \mathrm{~mm})$ with a protective precolumn $(50 \mathrm{~mm} \times 5 \mathrm{~mm})$ of Spherisorb ODS reverse-phase medium. The column was equilibrated with the same $0.05 \mathrm{M}$-sodium phosphate buffer $(\mathrm{pH} 1.7$ containing methanol $(100 \mathrm{ml} / \mathrm{l}))$ as was used to redissolve the samples after column chromatography. Samples were injected on to the column using a $\mathbf{R}$ heodyne syringe-loading injection valve with a $20 \mu \mathrm{lloop}$, and a single pump was used to elute materials isocratically at a flow rate of $1.5 \mathrm{ml} / \mathrm{min}$. Compounds in the eluate were detected by absorption at $265 \mathrm{~nm}$, using an $8 \mu 1$ flow cell in a Cecil CE-272 spectrophotometer attached to a Hewlett Packard 3390A recording integrator.

Preliminary studies showed good recovery of authentic standards of niacin metabolites through the HPLC procedure, with linearity over the range of $0 \cdot 015-1.0 \mathrm{~mm}$ concentrations 


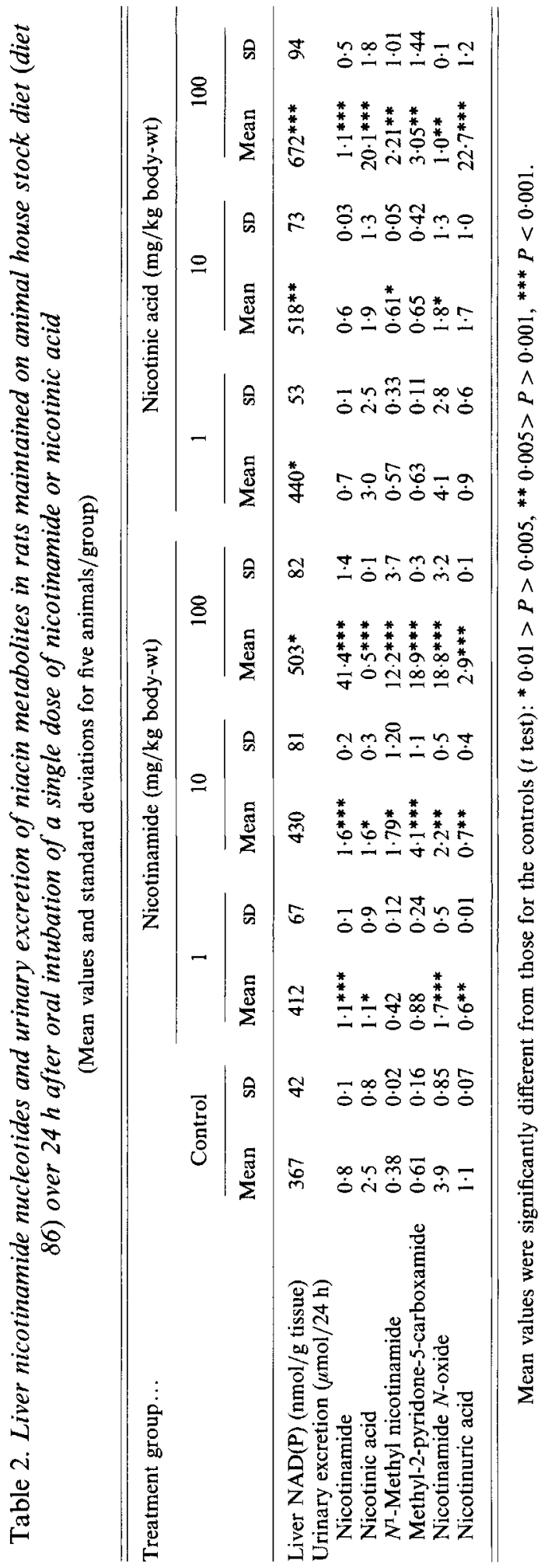


of all the metabolites measured. The retention times of the standards are shown in Table 1. Quinolinic acid could not be separated from the peak associated with the solvent front by this method. The limit of reliable detection was $1 \mathrm{pmol}$ injected onto the column for nicotinamide and $0.5 \mathrm{pmol}$ for the other metabolites studied. $N^{1}$-Methyl nicotinamide was not routinely measured by HPLC because it gave a broad diffuse peak, with a retention time of $33 \mathrm{~min}$.

There was one major peak of u.v. absorbing material in rat urine that increased with increasing intake or dose of niacin; it was in the neutral fraction and had a retention time of $8.0 \mathrm{~min}$. There were also a number of minor peaks. None of these has yet been identified, but preliminary studies suggest that none is a glucuronide or sulphate conjugate, since they were unaffected by incubation with a preparation of $\beta$-glucuronidase and sulphatase. It is likely that the major peak was methyl-4-pyridone-3-carboxamide, but no authentic sample of this was available to permit identification and quantification.

As observed previously (Bender et al. 1982), the animals fed on the diets providing tryptophan as the only precursor of nicotinamide nucleotides grew faster than those fed on low-tryptophan diets providing an adequate or more than adequate amount of nicotinamide or nicotinic acid, suggesting some degree of growth restriction by the low amount of tryptophan provided by these diets.

The liver content of NAD(P) and urinary excretion of niacin metabolites in animals receiving a single dose of nicotinic acid or nicotinamide are shown in Table 2 and, for animals receiving the six different diets described previously, in Table 3.

\section{DISCUSSION}

The acute dose of $10 \mathrm{mg}$ niacin $/ \mathrm{kg}$ body-weight is approximately equal to the daily intake of animals fed on diets providing $150 \mathrm{mg}$ niacin $/ \mathrm{kg}$ diet. However, this acute dose was superimposed on the relatively high amounts of nicotinic acid $(73 \mathrm{mg} / \mathrm{kg})$ and tryptophan $(2.4 \mathrm{~g} / \mathrm{kg})$ provided by the stock diet. It is therefore difficult to make direct comparisons between the results shown in Tables 2 and 3. Furthermore, it is to be expected that the metabolism of a single acute oral dose of niacin will differ from that of the same amount taken over the 4-6 h feeding period of the rat.

Neither nicotinic acid nor nicotinamide was excreted unchanged in substantial amounts in the diet study or after a single dose, except at the highest doses given $(100 \mathrm{mg} / \mathrm{kg}$ body-weight). This is in agreement with the results of Corr \& May (1975), who showed that nicotinic acid and nicotinamide are both actively resorbed from the glomerular filtrate, and will only be excreted in the urine when the concentration in the bloodstream is so high that the renal transport system is saturated.

In response to a single dose of nicotinic acid or a single high dose of nicotinamide $(100 \mathrm{mg} / \mathrm{kg}$ body-weight), there was a significant increase in the concentration of NAD(P) in the liver. Previous studies (Bender et al. 1982) have shown that nicotinate phosphoribosyltransferase $(E C 2.4 .2 .11)$ is not saturated at physiological concentrations of nicotinic acid, and therefore some increase in nucleotide synthesis might be expected with increasing availability of the substrate. However, nicotinamide phosphoribosyltransferase (EC 2.4.2.12) is saturated at physiological concentrations of its substrate. It is therefore unlikely that the increased concentration of nucleotides in the liver after a single high dose of nicotinamide is due to increased synthesis. Rather it is probable that it is the result of decreased catabolism; both nicotinamide and $N^{1}$-methyl nicotinamide, but not nicotinic acid, inhibit poly-(ADP-ribose) synthetase (NAD ${ }^{+}$ADP-ribosyltransferase, EC 2.4.2.30), one of the two enzymes involved in the catabolism of NAD (Berger $e t$ al. 1978; Levi $e t$ al. 1978; Purnell \& Whish, 1980). 
The excretion of $N^{1}$-methyl nicotinamide and methyl-2-pyridone-5-carboxamide increased with increasing doses of nicotinamide, and at high doses there was also an increase in the excretion of nicotinamide $N$-oxide. At high doses of nicotinic acid there was also a small increase in the excretion of $N^{1}$-methyl nicotinamide and methyl-2-pyridone-5-carboxamide. While this might suggest some increased synthesis and catabolism of NAD, it might also reflect amidation of nicotinic acid to nicotinamide; reversal of the reaction of nicotinamide deamidase (nicotinamidase, EC 3.5.1.19).

Nicotinuric acid excretion was increased not only after the administration of its precursor, nicotinic acid, but also, to a lesser extent, after the administration of $100 \mathrm{mg}$ nicotinamide $/ \mathrm{kg}$ body-weight. The kinetic properties of rat liver nicotinamide deamidase are such that this enzyme is saturated at the normal steady-state concentration of nicotinamide in liver (Bender et al. 1982). Therefore it is not likely that there will be any additional activity at very high concentrations of nicotinamide, unless there is an isoenzyme of the deamidase with a very high Michaelis-Menten constant which has not been reported to date. However, a number of intestinal micro-organisms have nicotinamide deamidase activity (Gadd \& Johnson, 1974; Johnson \& Gadd, 1974) and it is possible that following a large oral dose of nicotinamide, bacterial deamidation in the intestinal lumen is an important route of metabolism, leading to absorption and subsequent metabolism of nicotinic acid. Indeed, Johnson \& Gadd (1974) have suggested that intestinal deamidation is the normal major fate of dietary nicotinamide. The present results do not support this suggestion, since the urinary metabolites of nicotinic acid and nicotinamide were quantitatively different in both the acute study (Table 2) and the diet study (Table 3 ).

Osmond \& Hoffer (1962) suggested a possible metabolic mechanism to explain the claimed anti-schizophrenic action of high doses of nicotinamide (up to $20 \mathrm{~g} / \mathrm{d}$ for an adult; Hoffer et al. 1957; Hoffer, 1971), although the efficacy of such so-called 'mega-vitamin therapy' has been challenged by a number of controlled trials, reviewed by Ban (1974), which have failed to demonstrate any significant anti-schizophrenia action of nicotinamide. One of the theories of the biochemical basis of schizophrenia is that there is abnormal methylation of a neurotransmitter or metabolite to produce an endogenous psychotogen, chemically akin to some of the hallucinogenic drugs (Pollin et al. 1961). Osmond \& Hoffer (1962) suggested that nicotinamide might reduce this abnormal methylation by acting as a 'sink' for methyl groups and depleting tissue reserves of single carbon units. Kang-Lee et al. (1983) showed some reduction in the total choline concentration in the plasma and liver of rats fed on a choline-free diet, as the intake of nicotinamide was increased from 60 to $600 \mathrm{mg} / \mathrm{kg}$ body-weight per $\mathrm{d}$. While the majority of the niacin metabolites recovered in the present study were indeed methylated, the proportion of the dose of nicotinamide recovered as $N^{1}$-methyl nicotinamide and methyl-2-pyridone-5-carboxamide decreased as the intake increased. It is therefore considered unlikely that high doses of nicotinamide would result in any metabolically important depletion of tissue methyl group donors. This is confirmed by preliminary studies of the effects of feeding rats on choline- and methionine-deficient diets. Depletion of tissue pools of methyl donors would be expected to reduce the ratio, phosphatidylcholine:phosphatidylethanolamine in membrane phospholipids. However, there was no change in the ratio, phosphatidylcholine:phosphatidylethanolamine in response to a prolonged, very high intake of nicotinamide $(1500 \mathrm{mg} / \mathrm{kg}$ diet for 7 weeks) (Bender et al. 1987).

The results of the diet study provide some insight into the relative importance of tryptophan and preformed dietary niacin as precursors of the nicotinamide nucleotide coenzymes. Assuming that $30 \mathrm{mg}$ dietary tryptophan is equivalent to $1 \mathrm{mg}$ niacin, the usual equivalence for the rat (Krehl et al. 1946; Hankes et al. 1948), the high-tryptophan diet provided the equivalent of $196 \mathrm{mg}$ niacin $/ \mathrm{kg}$, almost the same as the high-niacin diets 4 
and $6(150 \mathrm{mg} / \mathrm{kg})$. However, comparing the results in Table 3 for diets 1 and 2 , it can be seen that an almost threefold increase in tryptophan intake resulted in a $2 \cdot 6$-fold increase in liver NAD(P), whereas a tenfold increase in dietary nicotinamide (diets 3 and 4) or nicotinic acid (diets 5 and 6 ) resulted in only a $50 \%$ increase in liver nucleotides. There was also a greater increase in the urinary excretion of three niacin metabolites, $N^{1}$-methyl nicotinamide, methyl-2-pyridone-5-carboxamide and nicotinuric acid in response to a threefold increase in tryptophan intake than to a tenfold increase in niacin intake. These results support the suggestion made previously (Bender et al. 1982) that the utilization of nicotinic acid and nicotinamide is limited by the phosphoribosyltransferases involved (see Fig. 1), which are more or less saturated at the normal steady-state concentrations of nicotinamide and nicotinic acid in the liver, while there is no such limitation of the incorporation into nucleotides of quinolinic acid arising from tryptophan metabolism. The considerable increase in urinary niacin metabolites in animals fed on the high-tryptophan diet suggests that control of tissue concentrations of NAD $(P)$ may be achieved largely by altering the rate of catabolism, as indicated by studies of the effects of hypoxia and hyperoxia (McCreanor \& Bender, 1983).

A part of this study was supported by a grant from ISFE, The International Foundation for Nutrition Education and Nutrition Research, Zurich. These studies formed part of a thesis submitted by G.M.M. and accepted by the University of London for the degree of PhD.

\section{REFERENCES}

Baggenstoss, A. H., Christenson, N. A., Berge, K. G., Baldus, W. P., Spiekerman, R. E. \& Ellefson, R. D. (1967). Mayo Clinic Proceedings 42, 385-399.

Ban, T. A. (1974). International Pharmacopsychiatry 9, 172-187.

Baron, D. N., Dent, C. E., Harris, H., Hart, E. W. \& Jepson, J. B. (1956). Lancet ii, 421-428.

Bartlett, P. C., Morris, J. G. \& Spengler, J. (1982). US Public Health Reports 97, 258-260.

Bender, D. A. (1980). Biochemical Pharmacology 29, 2099-2104.

Bender, D. A., Clifford, E., McCreanor, G. M. \& Smith, A. D. (1987). Proceedings of the Nutrition Society (In the Press).

Bender, D. A., Magboul, B. I. \& Wynick, D. (1982). British Journal of Nutrition 48, 119-127.

Berger, N. A., Weber, G. \& Kaicha, A. (1978). Biochimica et Biophysica Acta 519, 87-104.

Biehl, J. P. \& Vilter, R. W. (1954). Proceedings of the Society for Experimental Biology and Medicine 85, 389-395.

Blom, W., van den Berg, G. B., Huijmans, J. G. M. \& Sanders-Woudstra, J. A. R. (1985). Journal of Inherited Metabolic Diseases 8, Suppl. 2, 107-108.

Carpenter, K. J. \& Kodicek, E. (1950). Biochemical Journal 46, 421-426.

Carpenter, K. J. \& Lewin, W. J. (1985). Journal of Nutrition 115, 543-552.

Carter, E. G., Hurrell, R. F. \& Carpenter, K. J. (1977). Proceedings of the Nutrition Society 36, 107A

Castiello, R. J. \& Lynch, P. J. (1972). Archives of Dermatology 105, 574-577.

Corr, P. B. \& May, D. G. (1975). Journal of Pharmacology and Experimental Therapeutics 192, 195-200.

Gadd, R. E. A. \& Johnson, W. J. (1974). International Journal of Biochemistry 5, 397-407.

Hankes, L. V., Henderson, L. M., Brickson, W. L. \& Elvehjem, C. A. (1948). Journal of Biological Chemistry 174, $873-881$.

Harrison, R. J. \& Feiwel, M. (1956). British Medical Journal ii, 852--854.

Hoffer, A. (1971). Canadian Psychiatric Association Journal 16, 499-504.

Hoffer, A., Osmond, H., Callbeck, M. J. \& Kahan, I. (1957). Journal of Clinical and Experimental Psychopathology 18, $131-158$

Holman, W. I. M. \& Wiegand, C. (1948). Biochemical Journal 43, 423-426.

Johnson, W. J. \& Gadd, R. E. A. (1974). International Journal of Biochemistry 5, 633-641.

Kang-Lee, Y. A. E., McKee, R. W., Wright, S. M., Swendseid, M. E., Jenden, D. J. \& Jope, R. S. (1983). Journal of Nutrition 113, 215-221.

Kaplan, N. O., Colowick, S. P. \& Barnes, C. C. (1951). Journal of Biological Chemistry 191, 461-472.

Krehl, W. A., Sarma, P. S., Teply, L. J. \& Elvehjem, C. A. (1946). Journal of Nutrition 31, 85-106.

Lehman, J. (1972). Acta Physiologica Scandinavica Suppl. 237.

Levi, V., Jacobson, E. \& Jacobson, M. (1978). FEBS Letters 88, 144-146. 
Lowry, O. H., Passoneau, J. V. \& Rock, M. K. (1961). Journal of Biological Chemistry 236, 2756-2759.

McCreanor, G. M. \& Bender, D. A. (1983). Biochimica et Biophysica Acta 759, 222-228.

Magboul, B. I. \& Bender, D. A. (1983). British Journal of Nutrition 49, 321-324.

National Research Council (1962). Nutrient Requirements of Laboratory Animals, Washington DC: US Government Printing Office.

Osmond, H. \& Hoffer, A. (1962). Lancet i, 316-320.

Parsons, W. B. (1961 a). Archives of Internal Medicine 107, 639-652.

Parsons, W. B. (1961 b). Archives of Internal Medicine 107, 653-667.

Pollin, W., Cardon, P. V. \& Kety, S. S. (1961). Science 133, 104-105.

Purnell, M. R. \& Whish, W. J. D. (1980). Biochemical Journal 185, 775-777.

Salih, M. A. M., Bender, D. A. \& McCreanor, G. M. (1985). Pediatrics 76, 787-793.

Swain, C. P., Tavill, A. S. \& Neale, G. (1976). Gastroenterology 71, 484-487.

Winter, S. L. \& Boyer, J. L. (1973). New England Journal of Medicine 289, 1180-1182. 